\title{
A distributed computing approach to improve the performance of the Parallel Ocean Program (v2.1)
}

\author{
B. van Werkhoven ${ }^{1}$, J. Maassen ${ }^{2}$, M. Kliphuis ${ }^{3}$, H. A. Dijkstra ${ }^{3}$, S. E. Brunnabend ${ }^{3}$, M. van Meersbergen ${ }^{2}$, \\ F. J. Seinstra ${ }^{2}$, and H. E. Bal ${ }^{1}$ \\ ${ }^{1}$ VU University Amsterdam, Amsterdam, the Netherlands \\ ${ }^{2}$ Netherlands eScience Center, Amsterdam, the Netherlands \\ ${ }^{3}$ Institute for Marine and Atmospheric research Utrecht, Utrecht, the Netherlands
}

Correspondence to: B. van Werkhoven (ben@cs.vu.nl)

Received: 5 July 2013 - Published in Geosci. Model Dev. Discuss.: 12 September 2013

Revised: 17 December 2013 - Accepted: 2 January 2014 - Published: 7 February 2014

\begin{abstract}
The Parallel Ocean Program (POP) is used in many strongly eddying ocean circulation simulations. Ideally it would be desirable to be able to do thousand-yearlong simulations, but the current performance of POP prohibits these types of simulations. In this work, using a new distributed computing approach, two methods to improve the performance of POP are presented. The first is a blockpartitioning scheme for the optimization of the load balancing of POP such that it can be run efficiently in a multiplatform setting. The second is the implementation of part of the POP model code on graphics processing units (GPUs). We show that the combination of both innovations also leads to a substantial performance increase when running POP simultaneously over multiple computational platforms.
\end{abstract}

\section{Introduction}

Physical oceanography is currently undergoing a paradigm shift in the understanding of the processes controlling the global ocean circulation. Two factors have contributed to this shift: (i) the now about $20 \mathrm{yr}$ long record of satellite data and (ii) the possibility to simulate the ocean circulation using models which include processes on the Rossby deformation radius $(10-50 \mathrm{~km})$. Resolving this scale captures the instability processes that lead to ocean eddies which subsequently interact and affect the large-scale ocean flow (Vallis, 2006).

The level of realism (in relation to available observations) in simulating the ocean with high-resolution, strongly eddying models substantially increases compared to the low-resolution models in which the effects of eddies are parametrized. For example, it leads to a much better simulation of the different oceanic boundary currents, in particular the separation of the Gulf Stream in the Atlantic. Also, the degree to simulate the surface kinetic energy distribution, which can be compared with satellite data, markedly improves (Smith et al., 2000; Maltrud et al., 2010).

The use of the strongly eddying models is, even on the supercomputing platforms currently available, still computationally expensive, and simulations have a long turn-around time. Typical performances are from one to a few model years per $24 \mathrm{~h}$ using thousands of cores (Dennis, 2007). Considering the fact that it takes at least $1000 \mathrm{yr}$ to reach a nearstatistical-equilibrium state, innovations to increase the performance of these models and to efficiently analyse the data from the simulations have a high priority.

Today many traditional cluster systems are equipped with graphics processing units (GPUs) because of their ability to process computationally intensive workloads at unprecedented throughput and power efficiency rates. Existing software requires modifications such as the expression of finegrained parallelism before it may benefit from the added processing power that GPUs offer.

GPUs have been used to successfully accelerate numerical simulations before. For example, Michalakes and Vachharajani (2008) used GPUs to improve the performance of the Weather Research and Forecast (WRF) model. Similarly, Bleichrodt et al. (2012) implemented a numerical solver for the barotropic vorticity equation for a GPU. 
However, it is currently not well known which specific parts of ocean models can benefit the most from execution on GPUs, how the existing software should be revised to efficiently use GPUs, and what impact the use of GPUs will have on performance. In this paper, we aim to answer these questions.

We present two innovations to improve the performance of the Parallel Ocean Program (POP). POP is also used as the ocean component of the much used Community Earth System Model (CESM). We have applied our modifications to a standalone version of POP (v2.1). However, we have confirmed through source code inspection that all of our changes are also applicable to and fully compatible with the latest release of CESM (v1.2.0). The main issue is how to adapt POP such that it can run simultaneously (and efficiently) on multiple GPU clusters. First, we address alternative domain decomposition schemes and hierarchical load-balancing strategies which enable multi-platform simulations such that further scaling can be achieved. Second, we show how POP can be adapted to run on GPUs and study the effect of GPU usage on its performance. The source code of our modified version of POP can be obtained from https://github.com/NLeSC/eSalsa-POP/.

\section{Load balancing}

The model considered here is the global version of POP (Dukowicz and Smith, 1994) developed at Los Alamos National Laboratory. We consider the strongly eddying configuration, indicated by $R_{0.1}$, as used in recent high-resolution ocean model simulations (Maltrud et al., 2010; Weijer et al., 2012). This version has a nominal horizontal resolution of $0.1^{\circ}$ using a $3600 \times 2400$ horizontal grid with a tripolar grid layout, having poles in Canada and Russia. The model has 42 non-equidistant $z$ levels, increasing in thickness from $10 \mathrm{~m}$ just below the upper boundary to $250 \mathrm{~m}$ just above the lower boundary at $6000 \mathrm{~m}$ depth. In addition, bottom topography is discretized using partial bottom cells, creating a more accurate and smoother representation of topographic slopes.

\subsection{Domain decompositions and block distributions}

POP supports parallelism on distributed memory computers through the message passing interface (MPI). To distribute the computation over the processors, POP uses a threedimensional mesh, sketched in Fig. 1a. The domain is decomposed into equal-sized rectangular blocks in the horizontal direction. Each block also contains several layers in the vertical direction (depth). The blocks are then distributed over the available MPI tasks, where each task receives one or more blocks. Blocks consisting only of land points may be discarded from the computation. Below we will assume that a single MPI task is assigned to a processor core (unless stated otherwise).
Each block is surrounded by a halo region (Fig. 1b) that contains a copy of the information of the neighbouring blocks. These halos allow the calculations on each block to be performed relatively independently of its neighbour blocks, thereby improving parallel performance. Nevertheless, the data in the halo regions need to be updated regularly. This requires a data exchange between the blocks, which leads to communication between the MPI tasks, the amount of data depending on the width of the halo, the size of the blocks, and the block distribution over the MPI tasks.

In POP, the halo width is typically set to 2 . For an example block size of $60 \times 60$, the number of elements that need to be exchanged per block in every halo exchange is $4 \times(60 \times 2)+4 \times 4=496$. This number may need to be multiplied by the number of vertical levels, depending on the data structure on which the halo exchange is performed. Some data structures, like the horizontal velocity, store a value for every grid point at every depth level. As a result, a 3-D halo exchange is required that exchanges elements from every depth level. Others data structures, such as surface pressure, only consist of a single level. There, a 2-D halo exchange is sufficient.

For neighbouring blocks that are assigned to the same MPI task, the data exchange is implemented by an internal copy and no MPI communication is required. Also, no data need to be exchanged with (or between) land elements. Therefore, the amount of data that needs to be communicated between MPI tasks depends heavily on the way the blocks are distributed over the MPI tasks.

\subsection{Existing block-partitioning schemes}

POP currently supports three algorithms for distributing the blocks over the available MPI tasks, Cartesian, rake (Marquet and Dekeyser, 1998), and space-filling curve (Dennis, 2007). The Cartesian algorithm starts by organizing the tasks in a two-dimensional grid. Next, the blocks are assigned to these tasks according to their position in the domain. If the number of MPI tasks does not divide the number of blocks evenly in either dimension, some tasks may receive more blocks than others. In addition, some tasks may be left with less work (or even no work) if one or more blocks assigned to it only contain land. As shown in Dennis (2007), load imbalance between tasks can significantly degrade the performance of high-resolution ocean simulations.

The rake algorithm attempts to improve the load balance by redistributing the blocks over the tasks. Note that this requires that the number of blocks is significantly larger than the number of MPI tasks. The rake algorithm starts with a Cartesian distribution and the corresponding twodimensional MPI task grid. First, the average number of blocks per task is computed. Then, for each row in the task grid, the algorithm takes the first task in the row and determines whether the number of blocks exceeds the average. If so, the excess blocks are passed on to the next task. 

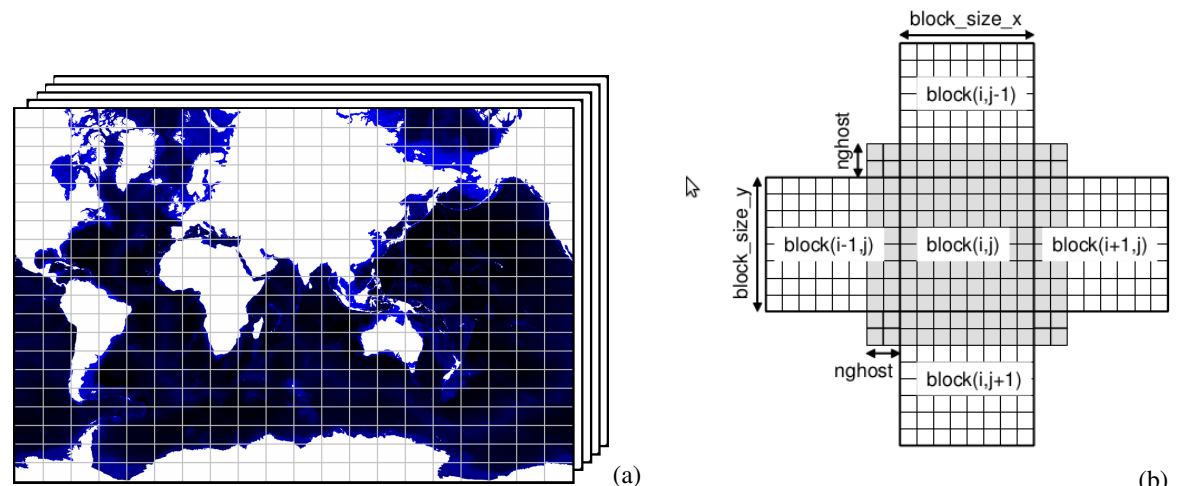

(a)

(b)

Fig. 1. (a) Sketch of the block-wise subdivision of the domain in POP. (b) The halo regions of a block; image from Smith et al. (2010).
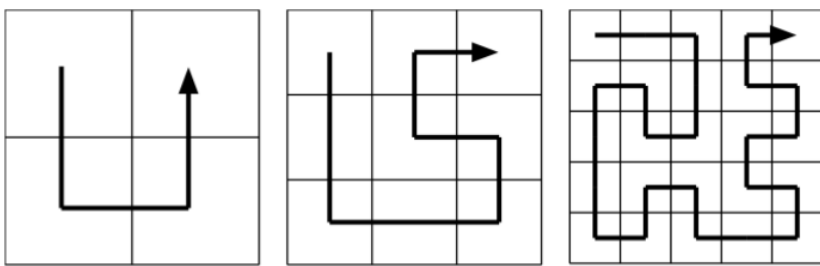

Fig. 2. Examples of the space-filling-curve load-balancing algorithm, with the Hilbert (left panel), meandering Peano (middle panel), and Cinco (right panel) curves; image from Dennis (2007).

This process is repeated for all tasks in the row. The process is repeated for all columns of the task grid. As described in Smith et al. (2010), the algorithm "can be visualized as a rake passing over each node and dragging excess work into the next available hole". In an attempt to keep neighbouring blocks close together, constraints are placed on block movements that prevent blocks from moving too far from their direct neighbours. Unfortunately, there are instances where the rake algorithm actually results in a worse load balance where blocks get raked into a corner. As a result Dennis (2007) states that "we do not consider the current implementation of the rake algorithm... sufficiently robust."

The space-filling-curve algorithm described in Dennis (2007) uses a combination of Hilbert, meandering Peano, and Cinco curves to partition the blocks (Fig. 2). Conceptually, it draws a single line that visits each of the blocks exactly once. It then splits this line into equal-sized segments, each segment visiting the same number of blocks. Due to the way the line is drawn, the blocks in each segment are also continuous in the two-dimensional domain. This solution degrades slightly when the land-only blocks are discarded, which introduces "cuts" in the curve. Nevertheless, the space-fillingcurve algorithm significantly improves the load balance between MPI tasks. A limitation of this approach is that each of the space-filling curves can only partition domains of a specific size. For example, a domain $P \times P$ can be partitioned by a Hilbert curve if $P=2^{n}$, or by a meandering Peano curve if $P=3^{m}$, where $n$ and $m$ are integers. By using combinations of different curves, the set of supported problem sizes can be extended.

\subsection{Hierarchical block partitioning}

None of the load-balancing algorithms described in the previous section takes into account the inherent hierarchical nature of modern computing hardware. This typically consists of multiple cores per processor, multiple processors per node, multiple nodes per cluster, and even the availability of multiple clusters for a numerical simulation. The communication performance drops as we go up in the hierarchy. The cores in a processor share cache memory and can therefore communicate almost instantaneously, while communication between processors has to go through main memory, which is much slower. Communication between processors on different nodes must go through an external network, which is orders of magnitude slower, and communication between clusters in different locations is again orders of magnitude slower. Therefore, simply balancing the load for the individual processors (or cores) is not sufficient. Instead, a hierarchical load-balancing scheme must be used that takes both processor load and the communication hierarchy of the target machine into account. We suggest using a similar approach to the one used in Zoltan (Zoltan User Guide, 2013; Teresco et al., 2005). However, where Zoltan supports dynamic load balancing (where the work distribution may change during the application's lifetime), we compute a single static solution before the application is started.

Our hierarchical load-balancing scheme, like the rake and space-filling-curve algorithms described earlier, assumes that the number of blocks is significantly larger than the number of processors. Instead of simply specifying the number of MPI tasks for which to create a partitioning, the user must now specify a sequence of partitionings. For example, a sequence $2: 16: 8$ indicates that the blocks must first be partitioned into 2 sets (preferably of equal size), each of which is 


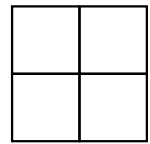

$[2,2]$

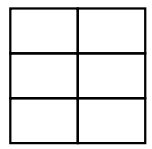

$[3,3]$

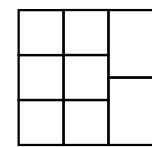

$[3,3,2]$

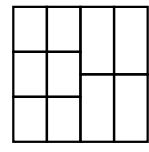

$[3,3,2,2]$
Fig. 3. Example subdivisions of a square into 4, 6, 8, and 10 rectangular sections.

then partitioned into 16 pieces, which are further divided into 8 pieces. The sequence of partitionings relates directly to the hierarchy that is present in the computational platform. For example, the $2: 16: 8$ partitioning can be used for an experiment on two clusters, each containing 16 nodes of 8 cores.

Once the user has specified the desired partitioning, the algorithm proceeds by repeatedly splitting the available blocks into $N$ (preferably equal-sized) subsets. We try to partition the domain in such a way that the shape of each of the subsets is as close to a square as possible. This will reduce the amount of communication out of each subset in relation to the amount of work inside each subset.

When splitting a domain, multiple solutions may be available which are equivalent from a load-balancing perspective. However, the amount of communication required between subsets may vary between these solutions due to assignment of blocks to MPI tasks and the location of land-only blocks. Our algorithm therefore compares these solutions and selects the one which generates the least communication between subsets.

To explain our algorithm in more detail, we use the simplified example domain shown in the upper left panel (a1) of Fig. 4. This example domain contains $1200 \times 1000$ grid elements. It is divided into blocks of $100 \times 100$, resulting in $12 \times 10$ blocks, of which 20 are land-only blocks. To divide this domain into 10 subsets, the algorithm starts by computing the required number of blocks per subset. The 100 nonland blocks must be divided into 10 subsets, resulting in 10 blocks per subset. Next, the algorithm tries to arrange the desired number of subsets in a (roughly) rectangular grid. The dimensions of this grid, consisting of $N$ subsets, is determined as follows:

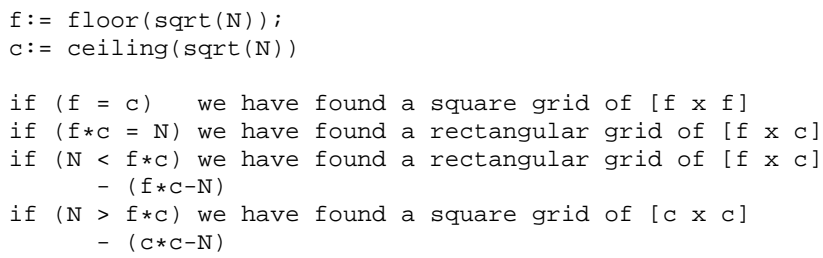

In the first two cases of the algorithm shown above, a square or rectangular decomposition is available containing exactly $N$ subsets. In the last two cases, the decomposition contains $(f * c-N)$ or $(c * c-N)$ subsets too many respectively. To correct this, we repeatedly remove a single subset from each row until the desired number of subsets is reached. Figure 3 shows four example subdivisions, for values of $N=4,6,8$, and 10, that correspond to each of these four cases. For our example domain we will use the rightmost subdivision in Fig. 3 for $N=10$ named [3,3,2,2], which represents the number of blocks in each column.

Next, we compute the required number of blocks per column using the average number of blocks per subset and the selected subdivision. For our example, we will use the $[3,3,2,2]$ subdivision as in Fig. 3 and the 10 blocks per subset average, which will result in columns containing $[30,30,20,20]$ blocks. We then split the domain into subsets by traversing the blocks in a vertical zigzag fashion and selecting all non-land blocks until the desired number of blocks for that column in reached. It should be noted that the partitioning scheme is not a flood-fill type of algorithm, which may skip over isolated points; instead, our partitioning scheme simply skips over any land points encountered while scanning in a certain direction, and continues scanning in a zigzag fashion until the required number of ocean (i.e. non-land) points have been selected.

The panels (a2-a6) in Fig. 4 show how the example domain is split into the four columns. We subsequently split each of the columns in a horizontal zigzag fashion into the desired number of subsets for that column. Panels b1-b5 of Fig. 4 show an example for the first column, which needs to be split into 3 subsets of 10 blocks. A similar subdivision is applied to the other columns. The final block distribution for the example domain is shown in Fig. 4c.

As explained above, the subdivision shown in panel (c) of Fig. 4 is only one out of a series of options. Several permutations of the $[3,3,2,2]$ subdivision can be created that are equivalent from a load-balancing perspective but require a different amount of communication. In addition, the subdivision can also be rotated, thereby initially dividing the domain row-wise instead of column-wise. Finally, when selecting the blocks in a zigzag fashion (as shown in Fig. 4), a choice can be made as to which position to start the selection from: top or bottom, or left or right. In our algorithm we simply compute all unique permutations of the subdivision in all possible rotations, with all possible starting points. We then select the solution with the lowest average communication per subset. If multiple equivalent solutions exist, we select the one with the lowest maximum communication per subset. Table 1 shows the best scoring results for all permutations of the $[3,3,2,2]$ subdivision. All solutions use the same number of blocks per task, but the amount of communication varies per solution. Once a domain has been split into the desired number of subsets, the algorithm is repeated for each of these subsets for the next split.

\subsection{Hierarchical partitioning of tripole grids}

In the application of the hierarchical load-balancing scheme to POP, the tripolar grid layout, where the North Pole is 
Step 1: Split domain column wise

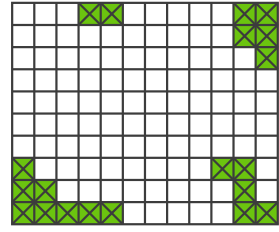

(a1): initial domain

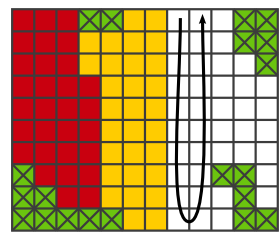

(a4): select 20 blocks for third column

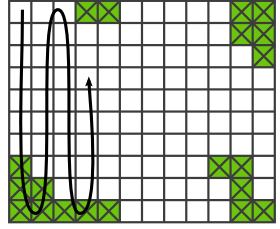

(a2): select 30 blocks for first column

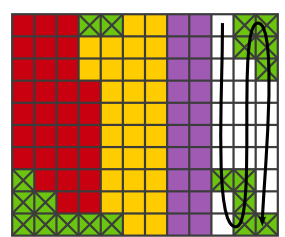

(a5): select 20 blocks for fourth column

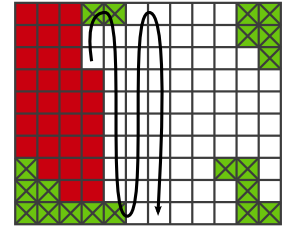

(a3): select 30 blocks for second column

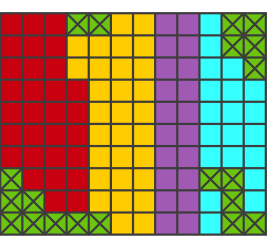

(a6): initial split completed

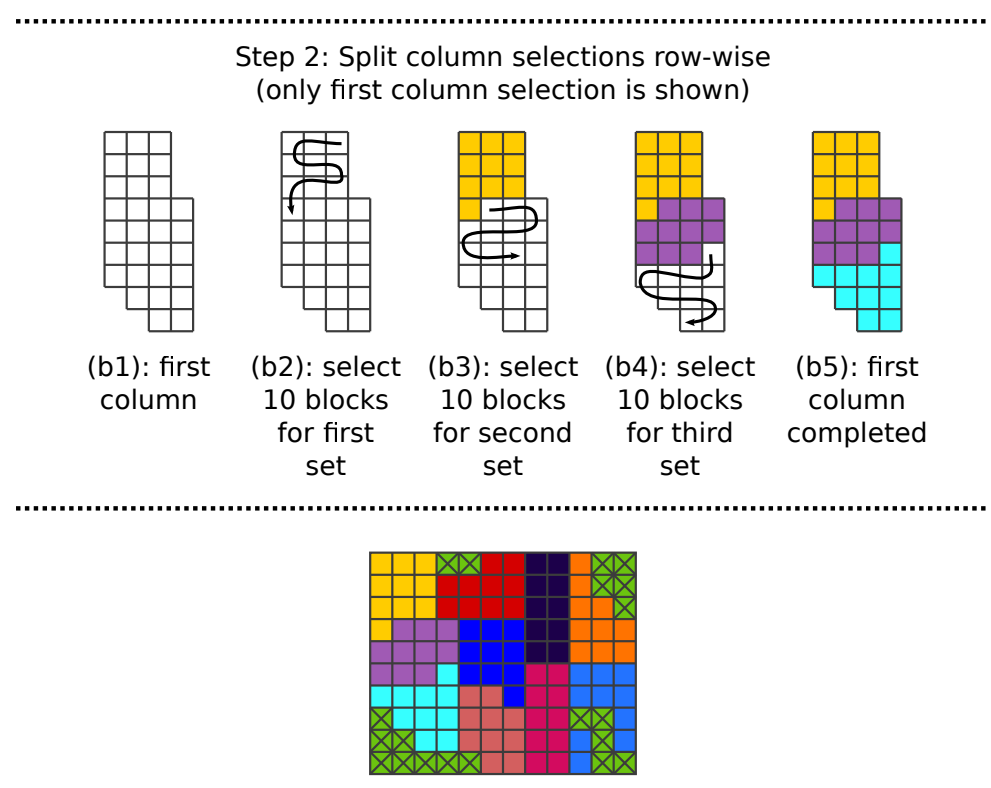

(c): final result

Fig. 4. Description of the hierarchical load-balancing scheme for an example of $12 \times 10$ blocks, of which 20 are land-only blocks, as shown in panel (a1). The initial column-wise split is shown in panels (a2)-(a6), the next row wise split in the panels (b1)-(b5), and the final results is shown in panel (c).

replaced with two poles located (on land) in Canada and Russia, needs special attention. Note that tripolar grids are frequently used in ocean models because the grid spacing in the Arctic is much more uniform and the cell aspect ratios are closer to 1 when compared to traditional latitude-longitude (dipole) grids (Smith et al., 2010). In this case, additional communication is required for the blocks located on the line between these poles, as explained in Smith et al. (2010). These blocks are located on the upper boundary of the grid, as shown in Fig. 5a. To support a tripolar grid layout in our hierarchical load-balancing scheme, we add the additional tripole communication to the communication requirements of the subset whenever a subset contains a tripole block. The extra communication will then be taken into account in the search phase of the algorithm. Although this approach will improve the partitioning, the result will not be optimal. As shown in Fig. 5a, two communicating tripole blocks may be located on opposite sides of the grid. This makes it difficult for our partitioning scheme to put these two blocks into the same subset. We overcome this problem by remapping 

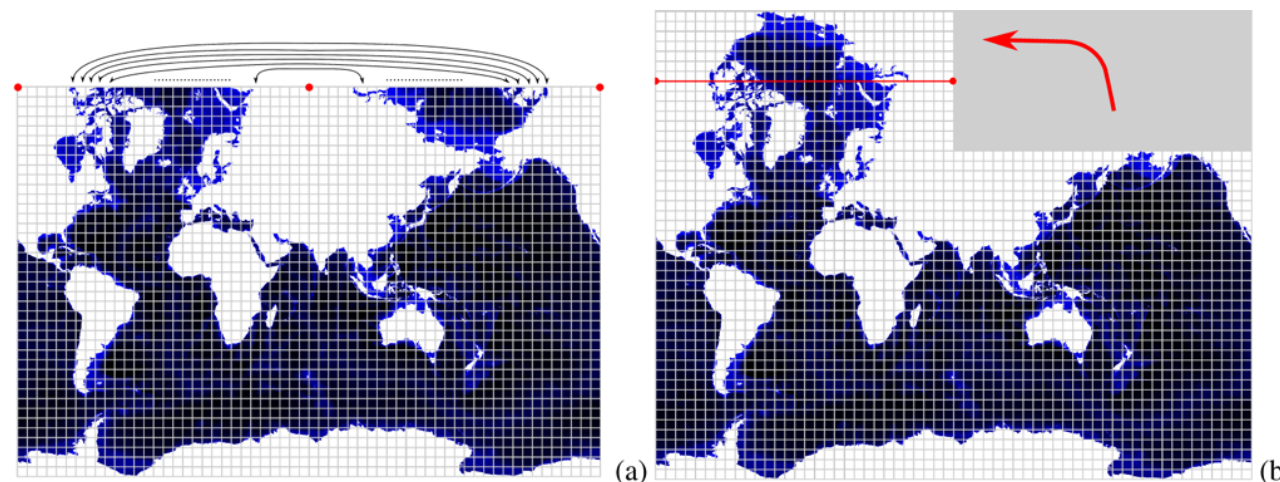

Fig. 5. (a) A subdivision of the topography into $60 \times 40$ blocks. The two tripoles are depicted by the red dots on the upper boundary. Note that the leftmost and rightmost dots represent the same tripole; the tripole communication is (partially) shown by the arrows. (b) A remapping of the grid that moves an area of $30 \times 7$ blocks. The original tripole boundary is shown as a red line.
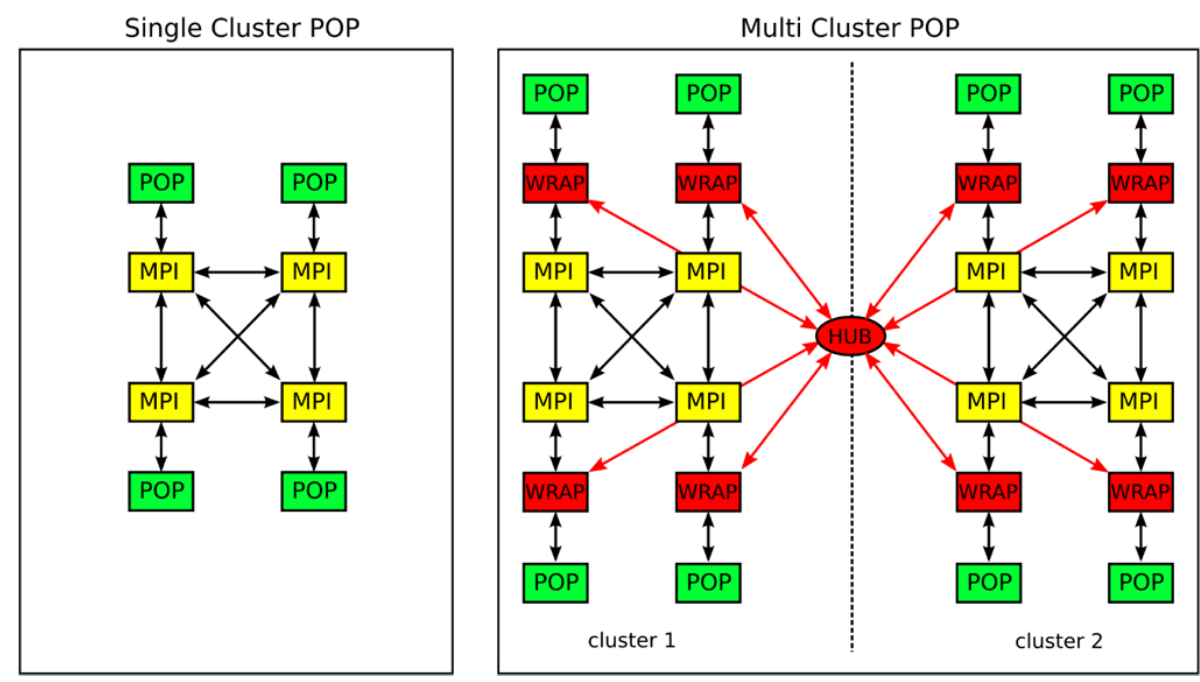

Fig. 6. An example of POP running without the MPI wrapper on a single cluster (left panel) and with the MPI wrapper on a multi-cluster (right panel).

Table 1. Permutations of the $[3,3,2,2]$ example distribution showing the number of assigned blocks and the communication per task in grid points per level. The entries are sorted by average communication per task. The topmost entry provides the best solution.

\begin{tabular}{lcc}
\hline permutation & $\begin{array}{c}\text { blocks } \\
\text { per task }\end{array}$ & $\begin{array}{c}\text { communication per task } \\
\text { (min/avg/max) }\end{array}$ \\
\hline$(3,3,2,2)$ & 10 & $1440 / 2186 / 2888$ \\
$(2,3,3,2)$ & 10 & $1244 / 2187 / 2888$ \\
$(2,2,3,3)$ & 10 & $1240 / 2188 / 3100$ \\
$(2,3,2,3)$ & 10 & $1240 / 2188 / 3300$ \\
$(3,2,2,3)$ & 10 & $1240 / 2229 / 3720$ \\
$(3,2,3,2)$ & 10 & $1440 / 2265 / 2876$ \\
\hline
\end{tabular}

the grid before we start the partitioning (Fig. 5b). By simply moving blocks from one side of the grid to the other, we enable our partitioning algorithm to optimize the tripole communication. Note that this remapping is only performed on the grid used in our partitioning algorithm. No change to POP is required, as POP only uses the result of the partitioning in which the original block numbering is maintained.

\section{Results: load balancing}

In this section we will compare the performance of our hierarchical algorithm to the Cartesian, rake, and space-fillingcurve block-partitioning schemes. In our experiments we carry out a 10-day simulation with the $R_{0.1}$ version of POP, as described at the beginning of Sect. 2, and show performance measures averaged over these 10 days. 


\subsection{Hardware}

The Huygens (http://www.surfsara.nl) is an IBM pSeries 575, a clustered SMP (symmetric multiprocessing) system. Each node contains 16 dual-core IBM Power 6 processors running at $4.7 \mathrm{GHz}$, resulting in 32 cores per node. As the cores support simultaneous multi-threading (SMT), every node appears to have 64 CPUs. Most applications will perform better by using 64 MPI tasks per node (two MPI tasks per processor core). Per node, $128 \mathrm{~GB}$ of memory is available (4 GB per core). The nodes are connected using $8 \times$ $\left(4 \times\right.$ DDR) InfiniBand, resulting in a $160 \mathrm{Gbit} \mathrm{s}^{-1}$ inter-node bandwidth.

The DAS-4 (http://www.cs.vu.nl/das4) is a six-cluster, wide-area distributed system. DAS-4 is heterogeneous in design, but in this experiment we will use dual quad-core compute nodes containing Intel E5620 CPUs running at $2.4 \mathrm{GHz}$, resulting in eight cores per node. The nodes contain $24 \mathrm{~GB}$ of memory (3 GB per core). Nodes are connected using QDR InfiniBand, resulting in a $20 \mathrm{Gbit} \mathrm{s}^{-1}$ bandwidth. We use DAS-4 in a single-cluster and two-cluster experiment. In the two-cluster experiment, the clusters are connected using a internet link with a maximum bandwidth of $1 \mathrm{Gbit} \mathrm{s}^{-1}$. The average round-trip time between clusters is $2.6 \mathrm{~ms}$. As the link is shared with other users, the available bandwidth and round trip latency may vary over time.

\subsection{Using MPI for multiple clusters}

For POP to run on multiple clusters, an MPI implementation is required that is capable of communicating both within and between clusters. This is far from trivial, as clusters are often protected by a firewall that disallows any incoming communication into the cluster. Also, it is common for the compute nodes to be configured such that they can only communicate with the cluster frontend, but not directly with the outside world, as explained in Maassen and Bal (2007). To solve this problem, we created wrapper code that is capable of intercepting the MPI calls in POP. For each intercepted call, the MPI wrapper decides whether it should be forwarded to the local MPI implementation or whether it should be sent to another cluster. To use the MPI wrapper code, POP needs to be recompiled using a different MPI library; however, no changes to the POP code itself are required.

To communicate between clusters, one or more support processes, so-called hubs, are used. Each hub typically runs on the cluster frontend, and serves as a gateway to the other clusters. If necessary, multiple hubs can be connected together to circumvent communication restrictions caused by firewalls. In Fig. 6, the left panel shows a traditional POP run on a single machine, while the right image illustrates how a hub is used in DAS-4 to connect two clusters together. Only a single hub is needed, as all compute nodes in DAS4 can communicate with all head nodes, even those of other

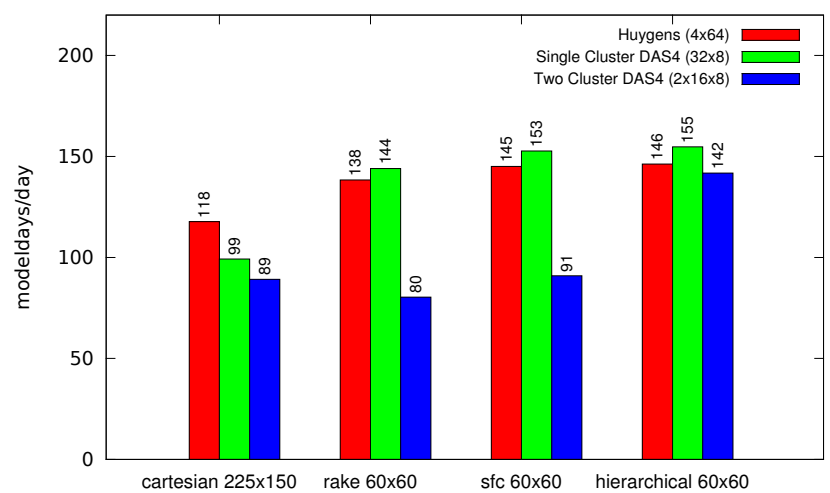

Fig. 7. Performance comparison of POP using Cartesian, rake, space-filling curve, and hierarchical block-partitioning schemes on three different hardware configurations, each using 256 MPI tasks.

clusters. However, compute nodes cannot directly communicate with compute nodes in other clusters.

\subsection{Performance}

Table 2 shows the configurations of the partitioning schemes. For each experiment we use 256 MPI tasks. The Cartesian distribution uses a $225 \times 150$ block size, resulting in exactly one block per MPI task (no land blocks are discarded). Both rake and the space-filling curve use a block size of $60 \times 60$ and discard 628 of 2400 blocks (i.e. $26 \%$ ). The table also shows the minimum, average, and maximum communication per MPI task, as well as the amount of traffic generated between the clusters for the two-cluster experiment. We will discuss these below. As can be seen from Table 2, the hierarchical domain distribution significantly decreases the amount of traffic between the clusters compared to rake and the space-filling curve. As a result, the performance overhead of using two clusters is limited.

The performance results of POP are shown in Fig. 7 in model day ${ }^{-1}$. On Huygens and single-cluster DAS-4, the rake and space-filling curve block distribution clearly improve the performance over the Cartesian distribution. On Huygens, the performance improvement of the space-filling curve is close to the amount of work discarded (23\% vs. $26 \%)$. On DAS-4 the improvement is much greater $(54 \%$ vs. $26 \%$ ) due to the better cache behaviour of smaller blocks. The space-filling curve distribution outperforms the rake distribution in all cases, due to the better load-balancing characteristics, as shown in Table 2. Figure 7 also shows that the performance degrades in the two-cluster DAS-4 experiments. Interestingly, the performance reduction for Cartesian is only $10 \%$, while the space-filling curve (41\%) and rake (44\%) are much more affected. This difference is caused by the increased communication caused by these two block distributions, as shown in Table 2. 
Table 2. Configuration of the Cartesian, rake, and space-filling curve, and hierarchical distributions.

\begin{tabular}{lccccc}
\hline algorithm & $\begin{array}{c}\text { block } \\
\text { size }\end{array}$ & $\begin{array}{c}\text { blocks } \\
\text { per core } \\
\text { (min/max) }\end{array}$ & $\begin{array}{c}\text { blocks } \\
\text { discarded }\end{array}$ & $\begin{array}{c}\text { communication } \\
\text { per task } \\
\text { (min/avg/max) }\end{array}$ & $\begin{array}{c}\text { communication } \\
\text { between clusters } \\
\text { (messages/volume) }\end{array}$ \\
\hline Cartesian & $225 \times 150$ & $1 / 1$ & 0 (of 256) & $0 / 1267.4 / 2408$ & $22.3 \mathrm{M} / 99.0 \mathrm{~GB}$ \\
rake & $60 \times 60$ & $5 / 8$ & 628 (of 2400) & $748 / 1940.5 / 3936$ & $77.9 \mathrm{M} / 337.4 \mathrm{~GB}$ \\
space-filling curve & $60 \times 60$ & $6 / 7$ & 628 (of 2400) & $1007 / 1707.7 / 2960$ & $41.0 \mathrm{M} / 212.7 \mathrm{~GB}$ \\
hierarchical & $60 \times 60$ & $6 / 7$ & 628 (of 2400) & $504 / 1394.9 / 2584$ & $20.0 \mathrm{M} / 82.5 \mathrm{~GB}$ \\
\hline
\end{tabular}

Table 3. Speed-up on DAS-4 for one- and two-cluster configurations using a hierarchical domain distribution.

\begin{tabular}{lcc}
\hline configuration & $\begin{array}{c}\text { performance } \\
\text { (modeldays day }^{-1} \text { ) }\end{array}$ & speed-up \\
\hline 1 cluster, 16 nodes & 82 & 1.0 \\
1 cluster, 32 nodes & 155 & 1.9 \\
2 clusters, 16 nodes each & 142 & 1.7 \\
\hline
\end{tabular}

Although rake and the space-filling curve both decrease the amount of work per MPI task, they also significantly increase the amount of communication between tasks. On supercomputers, where POP is traditionally run, this problem is mitigated by high-speed network interconnects, but in a multi-cluster environment, the internet link between clusters becomes a bottleneck. In Table 2, the column "communication between clusters" clearly shows that compared to Cartesian, rake causes an increase of 3.4 times in the communication between clusters. The increase caused by the spacefilling curve is smaller, a factor of 2.1, but still significant.

The hierarchical scheme performs slightly better than the space-filling curve scheme on Huygens and single-cluster DAS-4 (Fig. 7). This is to be expected, as the communication overhead is small on these systems due to the fast local network interconnects. On two-cluster DAS-4, however, the hierarchical domain distribution provides a significant performance improvement over the existing algorithms. When running on two clusters, the performance drop compared to a single-cluster run is only $8 \%$ for the hierarchical domain distribution, compared to $10 \%$ for Cartesian, $41 \%$ for the space-filling curve, and $44 \%$ for rake.

Table 3 shows the speed-up on DAS-4 compared to a 16node run on a single cluster. The speed-up on 32 nodes on a single cluster is, with a factor of about 1.9, almost perfect. Although the speed-up on two-clusters (of 16 nodes each) is slightly lower, about a factor of 1.7 , the performance gain compared to a single cluster is still significant. These results clearly demonstrate that using multiple clusters can be beneficial, especially to increase the number of machines beyond the size of a single cluster.

\section{Execution on GPUs}

This section discusses the main challenges that exist when moving parts of the computation in POP to a GPU. We use the CUDA programming model (Nvidia, 2013) in order to have fine-grained control over our GPU implementation and to be able to explain and improve performance results. Many different software tools, libraries, (directivebased) parallelization tools, and compilers aim to assist in the development of GPU code. However, it is our goal to gain a deep understanding of the performance behaviour of POP, which requires more control over the implementation and in particular how data are transferred between the host memory and GPU device memory. We are currently not aware of the capability to implement GPU kernels that overlap GPU computation with CPU-GPU communication in any of the existing directive-based parallelization tools for GPUs. However, if this were possible, it would require a collection of directives similar to the collection of calls to the CUDA runtime that are currently responsible for achieving this overlapping behaviour. While directive-based parallelization tools do leave the kernel code in the same language as the original, understanding the underlying architecture is still required in order to modify that parallelized code and assess its correctness. In the following sections, we use CUDA terminology (Nvidia, 2013), although our methods could just as easily apply to OpenCL (Khronos Group, 2013).

POP consists of a large Fortran 90 codebase, and in this paper we therefore limit ourselves to the most computeintensive parts of the program and only offload those computations to the GPU. The main challenge with this approach is to overcome the PCIe bus bottleneck. Whenever computations are to be performed on the GPU, the input and output data have to be transferred from host memory through the PCIe bus to GPU device memory and vice versa. The achieved bandwidth to GPUs connected through the PCIe 2.0 bus is approximately $5.7 \mathrm{~GB} \mathrm{~s}^{-1}$ from host to device and $6.3 \mathrm{~GB} \mathrm{~s}^{-1}$ from device to host. This is significantly lower than the bandwidth between host memory and a CPU and the bandwidth between GPU device memory and the GPU. Therefore, it is crucial that we maximize the overlap of data transfers to the GPU with computation and with transfers from the GPU back to the host. 
Table 4. List of the most compute-intensive functions in POP, covering $76.48 \%$ of the total computation time. The reported time does not include time spent in functions called by this function.

\begin{tabular}{lllll}
\hline \% time & function & module & \#calls & computes \\
\hline 15.09 & state & state_mod & 29562112 & density of water and derivatives \\
6.69 & hdiffu_del4 & hmix_del4 & 4865280 & horizontal diffusion of momentum \\
5.79 & advu & advection & 4865280 & advection of momentum \\
5.33 & bldepth & vmix_kpp & 115840 & ocean boundary layer depth \\
5.25 & hdifft_del4 & hmix_del4 & 4865280 & horizontal diffusion of tracers \\
4.62 & chrongear & pop_solversmod & 115840 & preconditioned conjugate-gradient solver \\
4.07 & ri_iwmix & vmix_kpp & 115840 & viscosity and diffusivity coefficients \\
3.83 & vmix_coeffs_kpp & vmix_kpp & 115840 & vertical mixing coefficients \\
3.66 & impvmixt_correct & vertical_mix & 115840 & implicit vertical mixing corrector step \\
3.34 & blmix & vmix_kpp & 115840 & mixing coefficients within boundary layer \\
3.27 & impvmixt & vertical_mix & 231680 & implicit vertical mixing of tracers \\
3.27 & clinic & baroclinic & 4865280 & forcing terms of baroclinic momentum \\
3.17 & advt_centered & advection & 4865280 & tracer advection using centred differencing \\
3.12 & btropoperator & pop_solversmod & 14705152 & applies operator for the barotropic solver \\
3.10 & baroclinic_driver & baroclinic & 115840 & integration of velocities and tracers \\
2.88 & ddmix & vmix_kpp & 115840 & add double-diffusion diffusivities \\
\hline
\end{tabular}

To overlap GPU communication and computation we need fine-grained control over how data are transferred to the GPU. There are several alternative techniques for moving data between host and device using the CUDA programming model. The most commonly used approach is to simply use explicit memory copy statements to transfer large blocks of memory to and from the GPU.

Alternatively, CUDA streams may be used to separate the computation into distinct streams that may execute in parallel. This way, communication from one stream can be overlapped with computation and communication in other streams. GPUs with 2 copy engines, such as Nvidia's Tesla $\mathrm{K} 20$, can use the PCIe bus in full duplex with explicit memory copies in different streams. This way, communication and computation from different streams can be fully overlapped.

Finally, the mapped memory approach uses no explicit copies, but maps part of the host memory into device memory space. Whether this approach is feasible depends on the memory access pattern of the kernel. Typically, mapped memory can only be used efficiently if each input and output element is read or written only once by the GPU function, called kernel. Although this approach results in very clean host code, requiring no explicit copy statements, it requires complex kernel implementations with intricate memory access patterns to ensure high performance.

\subsection{Targets for GPU implementation}

To determine which part of POP to port to the GPU, we must first get an impression of where the most time is spent. It is well known that the three-dimensional baroclinic solver is the most computationally intensive part of POP (Kerbyson and Jones, 2005; Worley and Levesque, 2003). We therefore limit ourselves to analysing the performance of the baroclinic solver.

Table 4 gives an overview of the most time-consuming functions in POP. These profiling results of are obtained from one month of simulation using the $R_{0.1}$ version (see beginning of Sect. 2) on the DAS-4 cluster (described in Sect. 2). For this experiment we have used a Cartesian distribution with blocks of size $255 \times 300$ and 8 processes per node on 16 nodes.

Table 4 lists the percentage of the total execution time spent in this function, not including subfunctions. All functions in Table 4, except those from the module pop solversmod, belong to the baroclinic solver. Our profiling results indicate that the baroclinic solver does not contain any true computational hotspots; that is, no individual function consumes a major part of the computation time.

However, the density computations from the equation of state are requested by several different parts both within the baroclinic solver and at the end of each time step. The computation of water densities is required so frequently by the model that their computation time consumes $15.09 \%$ of the total execution time on average.

The functions from the vmix_kpp module in Table 4 are part of the computation of the vertical mixing coefficients for the KPP mixing scheme (Large et al., 1994), which in total consumes about $35.3 \%$ of the total execution time. We therefore focus on obtaining a GPU implementation for the equation of state and for the computation of vertical mixing coefficients, in particular the three function states, buoydiff (the computation of buoyancy differences) and ddmix. We focus on buoydiff() and $\operatorname{ddmix}()$ since they are among the most compute intensive functions and are responsible for $64.9 \%$ of the calls to state(). 
It is well known that kernel-level optimizations focused on increasing computation throughput are generally not worthwhile when memory bandwidth is the primary factor in limiting performance (Ryoo et al., 2008). A frequently used tool for performance analysis on multi- and many-core hardware using the Roofline model (Williams et al., 2009) is the arithmetic intensity. For example, the Nvidia Tesla K20 GPU has a theoretical peak performance of $1173 \mathrm{GFLOP} \mathrm{s}^{-1}$ for double precision and a theoretical peak global memory bandwidth of $208 \mathrm{~GB} \mathrm{~s}^{-1}$. However, in practice the achieved memory bandwidth is (roughly) $160 \mathrm{~GB} \mathrm{~s}^{-1}$, as reported by the bandwidthTest tool in the Nvidia CUDA SDK. A rough estimation tells us that an arithmetic intensity of at least 7.3 FLOP byte ${ }^{-1}$ is required for the kernel to become compute-bound. Thus, if the arithmetic intensity is less than 7.3 FLOP byte ${ }^{-1}$, then we know the kernel is memorybandwidth-bound when executed on the K20.

The arithmetic intensity of the state() function is computed as follows. Although POP supports various implementations for the equation of state, we focus on the 25-term equation of state (McDougall et al., 2003) because it is the most commonly used implementation. The state() function requires the temperature and salinity tracers as inputs as well as 25 coefficients, of which 6 depend on the water pressure and the rest are constant. The state() function outputs the density of water and optionally also outputs the derivatives of the water density with respect to temperature and salinity. When only the density of water is computed, state() performs 40 floating point operations per grid point with an arithmetic intensity of 2.5 FLOP byte ${ }^{-1}$, assuming that all 25 coefficients can be stored in on-chip caches and can be fully reused. When all outputs are requested, 89 floating point operations are executed per grid point, resulting in an arithmetic intensity of 5.56 FLOP byte ${ }^{-1}$. With an arithmetic intensity of either 2.5 or 5.56 , the state() kernel is memory bandwidth-bound. Therefore, we focus on optimizing the time spent on communication between host and device rather than kernel-level optimizations.

\subsection{Efficient integration of GPU code}

We now describe how POP should be revised to efficiently use GPUs. For our discussion, we focus on three functions in POP state(), buoydiff(), and ddmix(). Due to a lack in GPU performance models that consider asynchronous PCIe transfers, it is currently impossible to predict what kind of implementation will be the most efficient. For each function we have therefore implemented three different versions that we call Explicit, Implicit, and Streams. We first describe the three versions in general and then discuss the specific implementations for state(), buoydiff(), and ddmix() in detail. Figure 8 provides a schematic overview of the three different implementations with regard to the way GPU computation (shown in green) and CPU-GPU communication (shown in blue) could be overlapped.

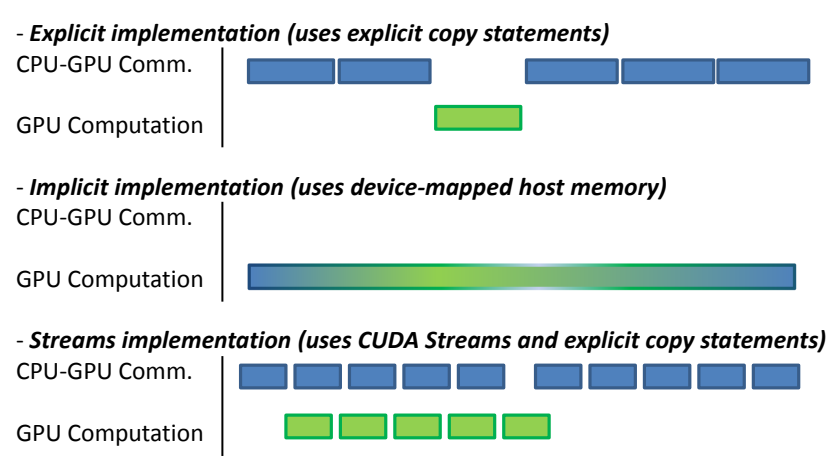

Fig. 8. Schematic of the three different implementations - Explicit, Implicit, and Streams - that shows the potential overlap between computation and communication.

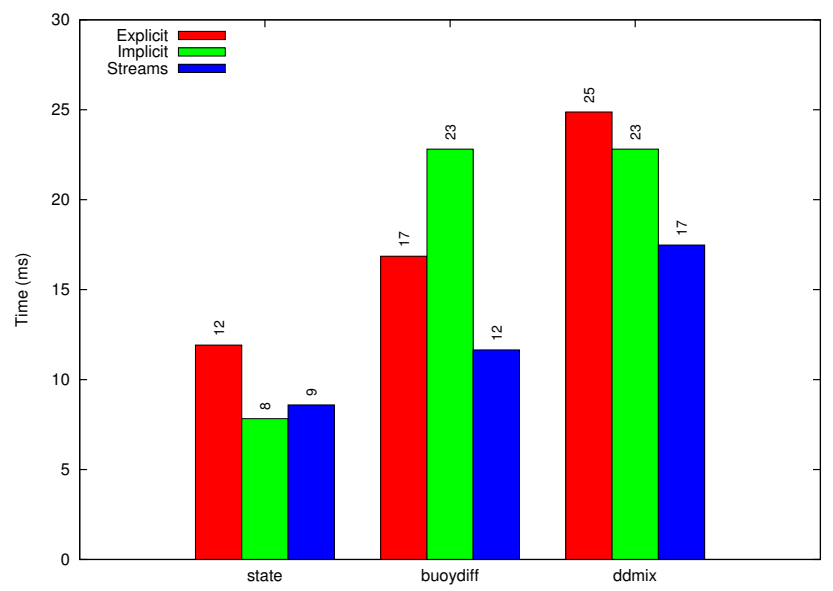

Fig. 9. Performance results for the three POP functions on a GPU with three different implementations as obtained on the Tesla K20 GPU with a $229 \times 304$ block size.

Explicit is a bulk-synchronous implementation that uses explicit memory copy statements to copy all the required input data to GPU and from the GPU for the entire threedimensional grid. The kernel used in Explicit creates a twodimensional array of threads, i.e. one thread for each horizontal grid point, which iterate the grid points in the vertical dimension. Implicit uses mapped memory and therefore requires no explicit memory copy statements. Instead, data are requested by the GPU directly from the host memory and sent over the PCIe bus. The performance of accessing the memory in this way is very sensitive to the order in which data are requested, and care must be taken not to create gaps or misalignments from the mapping between threads and data. Therefore, Implicit uses a kernel implementation that creates a one-dimensional array of threads with size equal to the number of grid points in the three-dimensional grid. Each thread then computes its three-dimensional index from its one-dimensional thread ID to direct itself to the correct part of the computation. The Streams implementation creates 
one stream for each vertical level and uses explicit copy statements to copy the corresponding vertical level of the input and output variables to and from the GPU. If the computation of one vertical level requires input from multiple vertical levels, CUDA events are used to delay the computation until all inputs have been moved to the device and vice versa. The kernel used in Streams is similar to the kernel used in Explicit except for the fact that the kernel only computes the grid points of one vertical level.

The three different implementations are very different in terms of code and the effort to create them. All three implementations use very distinctive host codes as well as modified GPU kernels. For example, the Implicit implementation barely requires any host code, whereas the Streams implementation requires multiple loops of memory copy operations and kernel invocations with advancing offsets. Note that, except for the differences described here, the kernels do not contain any architecture-specific optimizations.

While the state() function computes the density of water at a certain vertical level $k$, the function is mostly used directly surrounded by a loop over all vertical levels. These code blocks can safely be replaced by a call to a single function that directly computes the water densities for all vertical levels. Our Explicit implementation uses explicit copies to move the three-dimensional grid of tracer values between host and device and creates one thread for each horizontal grid point, which computes all outputs in the vertical direction. However, this approach is unable to overlap communication to and from the device with GPU computation. It is possible to also parallelize the computation of different vertical levels using CUDA streams. Our Streams implementation ensures that GPU computation can be overlapped with GPU communication of different vertical levels and thus alleviates the PCIe bus bottleneck to a large extent. Because of the simple access pattern in state(), where each input and output element is read or written only once, it is also a good candidate for the highly parallel Implicit implementation.

More complex uses of the equation of state are found within the computation of the vertical mixing coefficients for the KPP mixing scheme (Large et al., 1994), in particular in the computation of buoyancy differences (buoydiff) and double-diffusion diffusivities (ddmix). In POP the vertical mixing coefficients are sequentially computed for all vertical levels. The computation of buoyancy differences at level $k$ requires the density of both the surface level and level $k-1$ displaced to level $k$, as well as the water density at level $k$. These values can be computed for each level in parallel as long as all the data are present on the GPU. Overlapping data movement from the host to the GPU with GPU computation and data movement from the GPU to host becomes significantly more difficult, because the tracers for levels $1, k-1$, and $k$ need to be present on the GPU to compute the buoyancy differences at level $k$. The Streams implementation first schedules memory copies to the GPU for all vertical levels in concurrent streams and then invokes GPU kernel launches for all levels. However, before the execution of the kernel in stream $k$ can start, the memory copies in stream $1, k-1$, and $k$ need to be complete. The kernel executing in stream $k$ outputs to different vertical levels for different variables. Therefore, some of the memory copies from device to host in stream $k$ have to wait for the kernel in stream $k-1$ to complete. We use the CUDA event management functions to guarantee that no computations or memory transfers start prematurely.

In the ddmix function, the computation of diffusivities at level $k$ requires the derivatives of density with respect to temperature and salinity at level $k$ and $k-1$; that is, the computation of level $k$ reuses the derivatives that were used to compute level $k-1$. At a first glance, it would seem that the computation of all vertical levels cannot be parallelized. The sequential approach prevents these values having to be recomputed, but inhibits the ability to overlap communication and computation of different vertical levels. Therefore, our implementation also parallelizes the computation in the vertical dimension by introducing double work. The cost of computing the derivatives twice is significantly less than the inability to overlap computation and communication. Similarly to the buoyancy differences computation, the kernel executing in stream $k$ requires the memory copies of stream $k$ and $k-1$ to be complete. Again, CUDA event management functions are used to guarantee that no data are copied from the GPU back to the host before GPU computations have finished.

\section{Performance of POP on GPUs}

In this section, we will describe the performance of the $R_{0.1}$ version of POP on a single cluster and on multiple GPU clusters. In the first subsection below, we focus on the performance impact on individual POP subroutines when using a GPU. In the second subsection, we address the performance of the whole POP code on a single GPU and on multiple GPU clusters.

\subsection{Performance impact of GPU usage: individual routines}

First we evaluate the performance of single functions that were taken out of POP for individual benchmarking. We test our three implementations (Explicit, Implicit, and Streams) for each discussed function of POP on a single node equipped with a Nvidia Tesla K20 GPU in the DAS-4 cluster. The Tesla K20 has 2496 CUDA cores running at $705 \mathrm{MHz}$, providing a theoretical peak double-precision performance of $1173 \mathrm{GFLOP} \mathrm{s}^{-1}$. The K20 has $5 \mathrm{~GB}$ of device memory and a theoretical peak memory bandwidth of $208 \mathrm{~GB} \mathrm{~s}^{-1}$. The K20 is connected through a PCIe 2.0 bus and has two copy engines which enable full duplex use of the PCIe bus for concurrent explicit memory transfers. The grid dimensions used 
for the experiments discussed here are $229 \times 304 \times 42$. This is the same block size as used to obtain our profiling results, with two ghost cells in both horizontal dimensions. The performance results presented here are averaged execution times of five distinct runs. The execution times of these individual routines on the tested GPUs show minimal variance.

For all three implementations, most of the execution time is spent on transferring the data to and from the GPU. For example, for the Streams implementation of state() only $10.3 \%$ of the execution time is spent on GPU computation, and only 19.4 and $13.3 \%$ for buoydiff() and $\operatorname{ddmix}()$, respectively. Note that the reported times for buoydiff() and ddmix() include the time spent within state() when called as a subfunction. In fact, calls to state() from the GPU kernels of buoydiff() and ddmix() are inlined to optimize the data access pattern of these kernels.

Figure 9 shows the performance results for all three functions with three different GPU implementations. For the state() function the Implicit implementation provides the best performance. Although the kernel implementation used by Implicit is slightly less efficient than the kernel used by Explicit, the total execution time is significantly less because a large part of the memory transfers between host and device and computation is overlapped. While Streams achieves overlapping behaviour similar to Implicit, it is more coarsegrained, with one vertical level at a time rather than individual grid points. That explains why Implicit outperforms the Streams implementation for the state() function.

The buoydiff() function has a very low arithmetic intensity and therefore the computation again accounts for only a small part of the total execution time. The Implicit implementation is slower than Explicit because the access pattern in buoydiff() requires several input elements multiple times. As a result, the Implicit approach transfers more data than necessary over the PCIe bus. Although these transfers can be overlapped with computation and with transfers in the opposite direction, the performance penalty for transferring data multiple times reduces the overall performance. The Streams approach again benefits from the fact that data transfers and computation can be overlapped, but without the restrictions that come with the Implicit approach. The data access pattern in buoydiff() requires that operations in some streams may have to wait for operations in another stream to complete before they can start. The overhead of these synchronizations accounts for on average $3.26 \%$ of the total execution time of the Streams implementation.

To parallelize the computation of $\operatorname{ddmix}()$ in the vertical dimension, the Implicit and Streams implementations do some double work; that is, some values are computed twice by different threads operating at different vertical levels, whereas a thread in the Explicit approach may reuse that value from the computation of a previous vertical level. Therefore, the time spent in computation for Implicit and Streams is higher than that of Explicit. However, due to the overlap of computation and PCIe transfers in both directions, both Streams and Implicit do outperform the Explicit implementation in terms of total execution time. The Implicit implementation again suffers from the fact that, although overlapped with communication and computation, data have to be transferred multiple times through the PCIe bus.

In the GPU implementation of the POP we use in the next subsection, the Implicit implementation for state() and the Streams implementation for buoydiff() and ddmix() are used. As buoydiff() is executed before ddmix() as part of the computation of vertical mixing coefficients, $\operatorname{ddmix}()$ reuses the tracers that have been copied to the GPU by buoydiff(). Additionally, for all three functions, the execution on the GPU as well as all data transfers are overlapped with the computation of other functions on the CPU. Therefore, the CPU never has to wait for the results of GPU computations.

\subsection{Performance of POP on multiple (GPU) clusters}

In this section, we evaluate the performance of the combination of the two approaches presented in this paper. The goal of this evaluation is to assess whether the addition of a GPU is at all beneficial for performance on the application level. This is certainly not trivial, considering that large amounts of data have to be moved back and forth between the different memories over a relatively slow PCIe link. Additionally, only a small number of functions are executed on the GPU and a single GPU is shared between the various CPU cores. As such, we compare the performance of two versions of the program: one that only uses CPUs and one that uses the available CPUs as well as the GPU.

We recognize that a truly fair comparison between the different experimental setups is very hard to achieve. We take the achieved performance in terms of the number of model days per day of simulation as a measure for comparison. We have chosen not to normalize these results using additional metrics such as hardware costs or power consumption to keep the experimental setup as simple as possible. Hardware costs of both CPUs and GPUs are influenced by different factors in addition to their performance capabilities. Power consumption is an important factor in the operational costs for modern supercomputers. However, as only a small fraction of the code currently executes on the GPU, it is clear that with the current state of the software, the GPU will be idle for a large fraction of the execution. Whether a complete GPU implementation of POP is more efficient than a CPU-only implementation in terms of power consumption is an interesting issue, but it is outside the scope of this paper.

For this evaluation we use the DAS-4 cluster (described earlier in Sect. 3.1). First, eight compute nodes each containing two quad-core Intel E5620 CPUs (eight cores per node total) running at $2.4 \mathrm{GHz}, 24 \mathrm{~GB}$ of memory, and a Nvidia GTX480 GPU are used. In addition, we also use 8 compute nodes each containing two six-core Intel E5-2620 CPUs (12 cores per node total) running at $2.0 \mathrm{GHz}, 64 \mathrm{~GB}$ of memory, and a Nvidia Tesla K20 GPU each. As a reference for the 


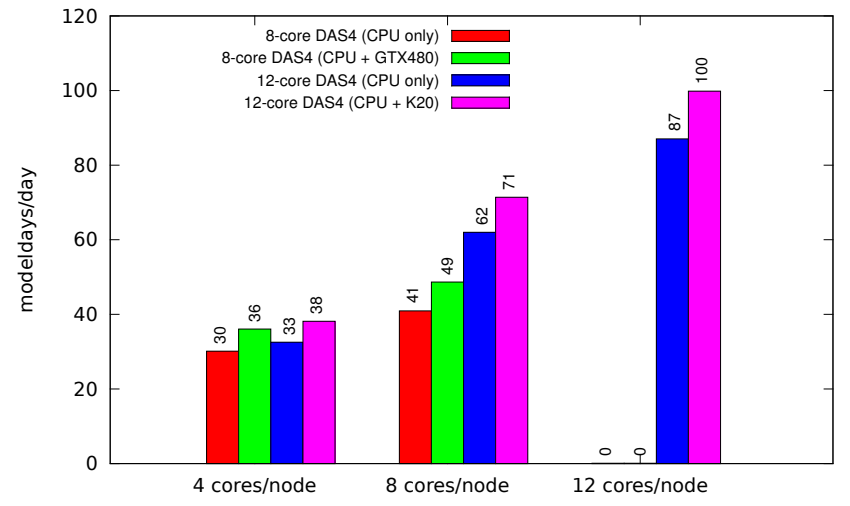

Fig. 10. Performance of POP using eight compute nodes of the DAS-4 cluster, with and without GPUs, using hierarchical partitioning with $60 \times 60$ block size.

CPU-only version of POP we use the original POP code with the hierarchical partitioning scheme described in Sect. 2.3. Comparisons against other load-balancing schemes can be derived from Fig. 7. All configurations in this section use a block size of $60 \times 60$.

Figure 10 shows the performance of POP using 4, 8, and 12 MPI tasks per node, with and without GPU. Note that only a single GPU is available in each node. Therefore, the GPU is shared between the multiple MPI tasks on a single node. For the eight-core DAS-4 nodes, the performance gained by using the GPU is approximately $20 \%$, both when using four or eight MPI tasks. This directly corresponds with the execution time consumed by POP code that has been ported to the GPU. The figure also shows that the scalability of POP itself is far from perfect. Running on eight MPI task per node, only provides a speed-up of 1.4 compared to four MPI tasks per node, both for the CPU-only and GPU versions.

For the 12-core DAS-4 nodes, the performance gained by using the GPU is approximately $15 \%$ when using 4 MPI tasks per node, and $13 \%$ when using 8 or 12 MPI tasks per node. Although this relative performance gain is lower that for the eight-core nodes, the absolute performance gain is much higher due to the better performance offered by the (newer) six-core CPU and K20 GPUs. In addition, the scalability of POP on the 12-core nodes is also much better, achieving a speed-up of 1.9 on 8 cores and 2.6 on 12 cores (both relative to the 4-core experiment).

The results show that it is possible to combine the hierarchical partitioning scheme with GPU execution and still obtain a performance increase. This is a remarkable result, as the hierarchical partitioning scheme prefers small block sizes, such as $60 \times 60$, to eliminate as many land-only blocks as possible and distribute load evenly among MPI tasks, while the GPU code would prefer larger-sized blocks to increase GPU utilization. However, GPU utilization is already increased by the fact that all MPI tasks running on a single node share a single GPU for all their GPU computations. It is

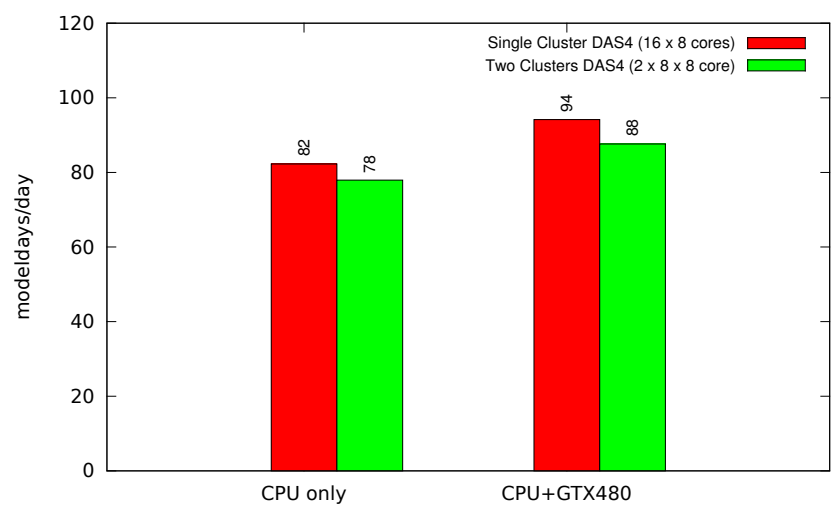

Fig. 11. Performance of POP using 16 compute nodes of the DAS-4 cluster, on one or two clusters, using hierarchical partitioning with $60 \times 60$ block size.

important to understand that this would not have been possible with larger block sizes because of the limited size of the GPU memory. As such, the two approaches presented in this paper work in concert to improve the performance of POP.

As a final experiment, we study the performance of POP on multiple platforms including GPUs. For this experiment, we use eight-core DAS-4 compute nodes with an Nvidia GTX480 GPU (described in Sects. 3.1 and 5.2).

Figure 11 compares the performance of a 16-node singlecluster run with a $2 \times 8$-node two-cluster run. Results are shown for CPU-only and CPU+GPU experiments. The results show a performance increase of $15 \%$ on one cluster and $13 \%$ on two clusters when using the GPUs. The performance loss when changing from one to two clusters is $5 \%$ for the CPU-only version and $6 \%$ for the CPU+GPU version. These results clearly indicate that running POP on multiple GPU clusters is feasible and also beneficial in terms of performance. Moreover, it allows users with access to multiple smaller GPU clusters to scale up to well beyond the size of a single GPU cluster.

\section{Summary, discussion, and conclusions}

High-resolution ocean and climate models are becoming a very important tool in climate research. It is crucially important that multi-century simulations with these models can be performed efficiently. In this paper, we presented a new distributed computing approach to increase the performance of the POP model.

First of all, we have shown that it is possible to optimize the load balancing of POP such that it can run successfully in a multi-platform setting. The hierarchical load-balancing scheme was shown to perform much better than the existing load-balancing schemes (Cartesian, rake, and space-filling curve), mainly due to the reduction in communication between the MPI tasks. In the future, we plan to take advantage 
of the Zoltan library in order to extend our load-balancing scheme so as to also take performance differences between machines into account. Secondly, it was demonstrated that it is advantageous to port part of POP to GPUs (and get a performance increase), even though POP itself does not contain any real hotspots and is therefore not an obvious candidate for using GPUs.

In the experiments shown, only three functions in POP were implemented on a GPU. Another substantial portion of the execution time is spent computing the advection of momentum and the horizontal diffusion of momentum and tracers. Obtaining a GPU implementation for these functions is deferred to future work. The advection of tracers also uses the equation of state to compute the potential density referenced to the surface layer, which is used to compute a variety of time-averaged fields. Currently, most of the execution time is spent on PCIe transfers. When more computation is moved to the GPU, more data can be reused, and some intermediate data structures that result from computation may even never have to leave the GPU. In that case, some PCIe transfers can be eliminated completely. In future work we hope to produce a complete GPU implementation of the vertical mixing part of POP.

The software presented in this paper has the same portability properties as the original POP. The GPU code is written in CUDA, which is a widely used language for GPU computing applications. To increase portability across different GPU architectures, no architecture-specific optimizations have been included. OpenCL is a well-known alternative to CUDA that aims at a wider set of many-core compute devices and different compilers are available for different platforms. However, there are no real linguistic differences between CUDA and OpenCL, and porting the code will be a simple engineering effort; furthermore, automated source-to-source translation tools are also available. The use of both extensions (domain decomposition or GPU functions) can be enabled, disabled, and controlled individually through the well-known pop_in namelist file.

Finally, we have shown that the combination of these two approaches also improves performance. Although we demonstrated this only for the DAS-4 cluster, it opens up the possibility to submit a POP job in the near future over multiple supercomputing platforms (with or without GPUs). The new hierarchical load-balancing scheme and the MPI wrapper methodology are crucial elements for maintaining the performance of POP. Future work is to port more of POP to GPUs and to scale up the multi-cluster experiments to production size hardware.

Acknowledgements. This publication is part of the eSALSA project (An eScience Approach to determine future Local Sea-level chAnges) of the Netherlands eScience Center (NLeSC), Institute for Marine and Atmospheric research Utrecht (IMAU) at Utrecht University, and VU University Amsterdam. This publication was supported by the Dutch national program COMMIT. Part of the computations were done on the Huygens IBM Power6 at SURFsara in Amsterdam (www.surfsara.nl). Use of these computing facilities was sponsored by the Netherlands Organisation for Scientific Research (NWO) under the project SH244-13. Support from NWO to cover the costs of this open access publication is also gratefully acknowledged.

Edited by: R. Redler

\section{References}

Bleichrodt, F., Bisseling, R., and Dijkstra, H. A.: Accelerating a barotropic ocean model using a GPU, Ocean Model., 41, 16-21, doi:10.1016/j.ocemod.2011.10.001, 2012.

Dennis, J. M.: Inverse space-filling curve partitioning of a global ocean model, IPDPS 2007, IEEE International, 1, 1-10, doi:10.1109/IPDPS.2007.370215, 2007.

Dukowicz, J. K. and Smith, R. D.: Implicit free-surface method for the Bryan-Cox-Semtner ocean model, J. Geophys. Res., 99, 7991-8014, doi:10.1029/93JC03455, 1994.

Kerbyson, D. J. and Jones, P. W.: A performance model of the parallel ocean program, Int. J. High Perform. C., 19, 261-276, doi:10.1177/1094342005056114, 2005.

Khronos Group: OpenCL, available at: http://www.khronos.org/ opencl/ (last access: August 2013), 2013.

Large, W. G., McWilliams, J. C., and Doney, S. C.: Oceanic vertical mixing: a review and a model with a nonlocal boundary layer parameterization, Rev. Geophys., 32, 363-403, doi:10.1029/94RG01872, 1994.

Maassen, J. and Bal, H. E.: Smartsockets: solving the connectivity problems in grid computing, in: Proceedings of the 16th IEEE International Symposium on High-Performance Distributed Computing (HPDC), Monterey, CA, USA, 1-10, doi:10.1145/1272366.1272368, 2007.

Maltrud, M., Bryan, F., and Peacock, S.: Boundary impulse response functions in a century-long eddying global ocean simulation, Environ. Fluid Mech., 10, 275-295, doi:10.1007/s10652009-9154-3, 2010.

Marquet, C. P. and Dekeyser, J. L.: Data-parallel load balancing strategies, Parallel Comput., 24, 1665-1684, doi:10.1016/S01678191(98)00049-0, 1998.

McDougall, T. J., Jackett, D. R., Wright, D. G., and Feistel, R.: Accurate and computationally efficient algorithms for potential temperature and density of seawater, J. Atmos. Ocean. Tech., 20, 730-741, doi:10.1175/15200426(2003)20<730:AAACEAF>2.0.CO;B2, 2003.

Michalakes, J and Vachharajani, M: GPU acceleration of numerical weather prediction, in: Proceedings of the International Symposium on Parallel and Distributed Processing (IPDPS), IEEE, 1-7, 2008.

Nvidia: CUDA Programming Guide, available at: http://docs. nvidia.com/cuda/ (last access: August 2013), 2013.

Ryoo, S., Rodrigues, C. I., Stone, S. S., Baghsorkhi, S. S., Ueng, S.Z., Stratton, J. A., and Hwu, W.-M. W.: Program optimization space pruning for a multithreaded GPU, in: Proceedings of the 6th Annual IEEE/ACM International Symposium on Code Generation and Optimization, ACM, doi:10.1145/1356058.1356084, 195-204, 2008. 
Smith, R. D., Maltrud, M. E., Bryan, F. O., and Hecht, M. W.: Numerical simulation of the North Atlantic Ocean at $\frac{1}{10}^{\circ}$, J. Phys. Oceanogr., 30, 1532-1561, 2000.

Smith, R., Jones, P., Briegleb, B., Bryan, F., Danabasoglu, G., Dennis, J., Dukowicz, J., Eden, C., Fox-Kemper, B., Gent, P., Hecht, M., Jayne, S., Jochum, M., Large, W., Lindsay, K., Maltrud, M., Norton, M., Peacock, S., Vertenstein, M., and Yeager, S.: The Parallel Ocean Program (POP) Reference Manual: Ocean Component of the Community Climate System Model (CCSM), 2010.

Teresco, J. D., Faik, J., and Flaherty, J. E.: Resource-aware scientific computation on a heterogeneous cluster, Comput. Sci. Eng., 7, 40-50, doi:10.1109/MCSE.2005.38, 2005.

Vallis, G. K.: Atmospheric and Oceanic Fluid Dynamics: Fundamentals and Large-Scale Circulation, Cambridge University Press, Cambridge, UK, 2006.
Weijer, W., Maltrud, M. E., Hecht, M. W., Dijkstra, H. A., and Kliphuis, M. A.: Response of the Atlantic Ocean circulation to Greenland Ice Sheet melting in a strongly-eddying ocean model, Geophys. Res. Lett., 39, L09606, doi:10.1029/2012GL051611, 2012.

Williams, S., Waterman, A., and Patterson, D.: Roofline: an insightful visual performance model for multicore architectures, Commun. ACM, 52, 65-76, doi:10.1145/1498765.1498785, 2009.

Worley, P. and Levesque, J.: The performance evolution of the parallel ocean program on the Cray X1, in: Proceedings of the 46th Cray User Group Conference, 17-21, 2003.

Zoltan User Guide: Hierarchical Partitioning, available at: http: //www.cs.sandia.gov/Zoltan/ug_html/ug_alg_hier.html (last access: December 2013), 2013. 